\title{
Enhanced oxidation resistance of MoTaTiCrAl high entropy alloys by removal of Al
}

\author{
Li-Chao Li ${ }^{1,2}$, Ming-Xing Li ${ }^{1,3}$, Ming Liu ${ }^{1,6}$, Bo-Yang Sun ${ }^{1,2}$, Chao Wang ${ }^{1}$, Jun-Tao Huo ${ }^{1,5,7}$, \\ Wei-Hua Wang ${ }^{1,3,4,5}$ and Yan-Hui Liu ${ }^{1,3,4,5^{*}}$
}

\begin{abstract}
Refractory high-entropy alloys (HEAs) exhibit remarkable mechanical properties desired for high-temperature applications. However, their oxidation resistance at high temperatures received less attention. Recent work indicates that MoTaTiCrAl alloy exhibits excellent oxidation resistance, but the effects of $\mathrm{Al}$ and $\mathrm{Cr}$ remain unclear. In this work, we demonstrate that the addition of $\mathrm{Al}$ is unnecessary for preventing oxidation of the MoTaTiCrAl alloy and the removal of Al leads to a more oxidation resistant MoTaTiCr medium entropy alloy. Structural and chemical analyses indicate that the excellent oxidation resistance of MoTaTiCr is mainly associated with the formation of continuous $\mathrm{CrTaO}_{4}$ oxide layer. The results indicate that complex oxides can be adopted as effective candidates for enhancement of oxidation resistance, in addition to typical strategy of forming $\mathrm{Al}_{2} \mathrm{O}_{3}, \mathrm{Cr}_{2} \mathrm{O}_{3}$ or $\mathrm{SiO}_{2}$ barrier layer.
\end{abstract}

Keywords: high-entropy alloy, oxidation resistance, MoTaTiCr, high-temperature application

\section{INTRODUCTION}

Oxidation resistance is critical for high-temperature applications of structural alloys because the formation of oxides can lead to severe degradation of mechanical properties [1]. From conventional perspective of alloy design, the primary strategy to enhance oxidation resistance is to incorporate $\mathrm{Al}, \mathrm{Cr}$ or $\mathrm{Si}$, as these elements are expected to form stable and dense protective oxide layers (e.g., $\mathrm{Al}_{2} \mathrm{O}_{3}, \mathrm{Cr}_{2} \mathrm{O}_{3}, \mathrm{SiO}_{2}$ ) that can prevent the diffusion of $\mathrm{O}^{2-}$ into the interior of alloys, leading to further oxidation [1-3]. However, traditional alloys are typically composed of one or two principal elements, with minor amount of other elements added to tailor microstructures and properties. Along with such design strategy, only a small fraction of $\mathrm{Al}, \mathrm{Cr}$, or $\mathrm{Si}$ is added for improving the oxidation resistance. The development of high-entropy alloys (HEAs), which are composed of five or more elements in (near) equimolar compositions, provides a new strategy for designing high-temperature alloys [4-8]. Instead of the formation of complex intermetallic phases, many HEAs form solid solutions in a wide compositional range and exhibit excellent phase stability, such that tuning of compositions and thus properties becomes possible in a vast range [4-8]. This novel strategy for alloy design not only significantly increases the number of potential alloys for technological applications, but also represents a new perspective for the development of highly oxidation-resistant alloys because a larger amount of $\mathrm{Al}, \mathrm{Cr}$, or $\mathrm{Si}$ can be incorporated into these alloy systems without sacrificing their mechanical properties [9].

Refractory HEAs (RHEAs), containing elements of high melting temperatures, such as $\mathrm{Mo}, \mathrm{Ta}, \mathrm{W}, \mathrm{Nb}, \mathrm{Hf}$, are demonstrated to possess excellent mechanical properties at both room and elevated temperatures [4,10,11]. Such properties imply that they are promising candidates for the applications as high-temperature structural alloys. Nonetheless, compared with the investigation on their mechanical properties, oxidation resistance of RHEAs received less attention [12]. Recently, (Mo, W)-(Nb, Ta, $\mathrm{Ti}, \mathrm{Zr}, \mathrm{Hf})-(\mathrm{Cr}, \mathrm{Al}, \mathrm{Si})$ alloys were found to exhibit pro-

\footnotetext{
${ }^{1}$ Institute of Physics, Chinese Academy of Sciences, Beijing 100190, China

${ }^{2}$ School of Physical Science, University of Chinese Academy of Sciences, Beijing 100049, China

${ }^{3}$ Songshan Lake Materials Laboratory, Dongguan 523808, China

${ }^{4}$ Beijing Advanced Innovation Center for Materials Genome Engineering, University of Science and Technology Beijing, Beijing 100083, China

${ }^{5}$ Center of Materials Science and Optoelectronics Engineering, University of Chinese Academy of Sciences, Beijing 100049, China

${ }^{6}$ Qian Xuesen Laboratory of Space Technology, Beijing 100094, China

${ }^{7}$ Ningbo Institute of Materials Technology and Engineering, Chinese Academy of Sciences, Ningbo 315201, China

* Corresponding author (email: yanhui.liu@iphy.ac.cn)
} 
mising oxidation resistance in the temperature range of $900-1100^{\circ} \mathrm{C}$. Among the alloys, MoTaTiCrAl was reported to be one of the most oxidation-resistant HEAs [12-16]. Analyses on the oxide layer indicate that the formation of a complex $\mathrm{CrTaO}_{4}$ oxide plays an important role in the enhanced oxidation resistance of the alloy system, because a dense $\mathrm{CrTaO}_{4}$ layer can inhibit outward diffusion of the constituent elements [13-15]. Although HEAs typically refer to alloys having at least five elements, suitable selection of elements is more important than solely increasing the number of elements in designing RHEA $[9,15,17]$.

In this study, we demonstrate that MoTaTiCr, the derivative of MoTaTiCrAl RHEA, exhibits better oxidation resistance. By evaluating the oxidation behavior of MoTaTi, MoTaTiAl, MoTaTiCr, and MoTaTiCrAl, we found that although the addition of $\mathrm{Al}$ into MoTaTi can result in increased oxidation resistance, the Al-free alloy MoTaTiCr was more resistant to oxidation as characterized by the smallest mass change and thinnest oxide layer during isothermal exposure at high temperatures in air. It was found that $\mathrm{CrTaO}_{4}$ with larger Pilling-Bedworth ratio (PBR) can serve as a better protective oxide layer, according to the observation that Mo oxide evaporated at high temperatures, leading to porous oxide layer.

\section{EXPERIMENTAL SECTION}

As the elements used in this study have significantly different melting temperatures, in order to guaranty chemical homogeneity of the fabricated samples, the equimolar MoTaTi, MoTaTiAl, MoTaTiCr, and MoTaTiCrAl alloys were produced by powder compaction followed by arc melting the powder tablets. Pure metal powders were accurately weighted and fully mixed at equal atomic ratios. For safety, small Al pellets, instead of Al powder, were used. The purity of all metal powders and $\mathrm{Al}$ pellets is more than $99.9 \%$. The mixed powders were placed in a tablet mold and compacted at room temperature under a pressure of $300 \mathrm{MPa}$ for $3 \mathrm{~min}$. The tablets were then arc-melted in Ar atmosphere using a water-cooled copper crucible. The ingots were flipped over and remelted for at least four times to ensure chemical homogeneity. Samples with a dimension of $2.5 \mathrm{~mm}$ $\times 2.5 \mathrm{~mm} \times 1 \mathrm{~mm}$ were cut from the ingots and polished by using P300 to P5000 SiC papers. The crystal structures and microstructures were characterized by using $\mathrm{X}$-ray diffraction (XRD) (Bruke D8) and scanning electron microscopy (SEM) (Phenom XL), respectively. Chemical analysis was carried out by using energy-dispersive $\mathrm{X}$-ray (EDX) spectrum attached to SEM. Oxidation tests were conducted by exposing the samples in air for $10 \mathrm{~h}$ at temperatures ranging from 500 to $1000^{\circ} \mathrm{C}$. Quantitative evaluation of oxidation resistance was performed by using a thermogravimetric analyzer (TGA) (STA 449F3) to measure the mass change as a function of isothermal exposure time, during which the samples were heated at a rate of $20^{\circ} \mathrm{C} / \mathrm{min}$ from room temperature to $1000^{\circ} \mathrm{C}$ and held at $1000^{\circ} \mathrm{C}$ for $5 \mathrm{~h}$ in air.

\section{RESULTS AND DISCUSSION}

\section{Microstructure of as-cast alloys}

Fig. 1a presents the XRD patterns of the as-cast MoTaTi, MoTaTiAl, MoTaTiCr, and MoTaTiCrAl alloys. For each alloy, the patterns exhibit only one set of diffraction peaks, which are identified to be a single-phase solid solution having a body-centered cubic (BCC) structure. This is expected because their physical and thermal parameters, such as atomic size difference, mixing enthalpy, and mixing entropy (Table 1), are all within the previously proposed range for the formation of solid solution $[18,19]$. With the addition of $\mathrm{Al}$ and $\mathrm{Cr}$, the Bragg peaks shift towards higher diffraction angle, indicating the shrinkage of lattice parameters that are calculated to be $0.323,0.321,0.319$, and $0.318 \mathrm{~nm}$ for MoTaTi, MoTaTiAl, MoTaTiCr, and MoTaTiCrAl, respectively. The overall compositions of the alloys are confirmed to be close to equimolar ratio (Table 2). Microstructures of the as-cast alloys are shown in Fig. 1b-e. As can be seen, they all exhibit dendrite microstructures. The average sizes of the dendrite arms are 40, 7, 20, $6 \mu \mathrm{m}$ for MoTaTi, MoTaTiAl, MoTaTiCr, and MoTaTiCrAl, respectively. The formation of the dendritic microstructures can be attributed to non-equilibrium solidification [20]. Chemical analyses indicate that the bright dendritic core is rich in elements of higher melting temperatures such as Ta and Mo, while the dark interdendritic region is rich in elements such as $\mathrm{Al}, \mathrm{Cr}$ and $\mathrm{Ti}$ that have lower melting temperatures (Table 2).

\section{Oxidation resistance}

The oxidation resistance of the alloys was first qualitatively evaluated by observing the changes in sample appearance upon isothermal exposure for $10 \mathrm{~h}$ at temperatures ranging from 500 to $1000^{\circ} \mathrm{C}$ in air. Fig. $2 \mathrm{a}$ shows the evolution of appearance for the samples with increasing exposure temperature. For ternary MoTaTi alloy, the sample turns black at $700^{\circ} \mathrm{C}$, suggesting obvious oxidation. With the increase of exposure temperature, the shape of sample gradually turns to be rounded and finally 

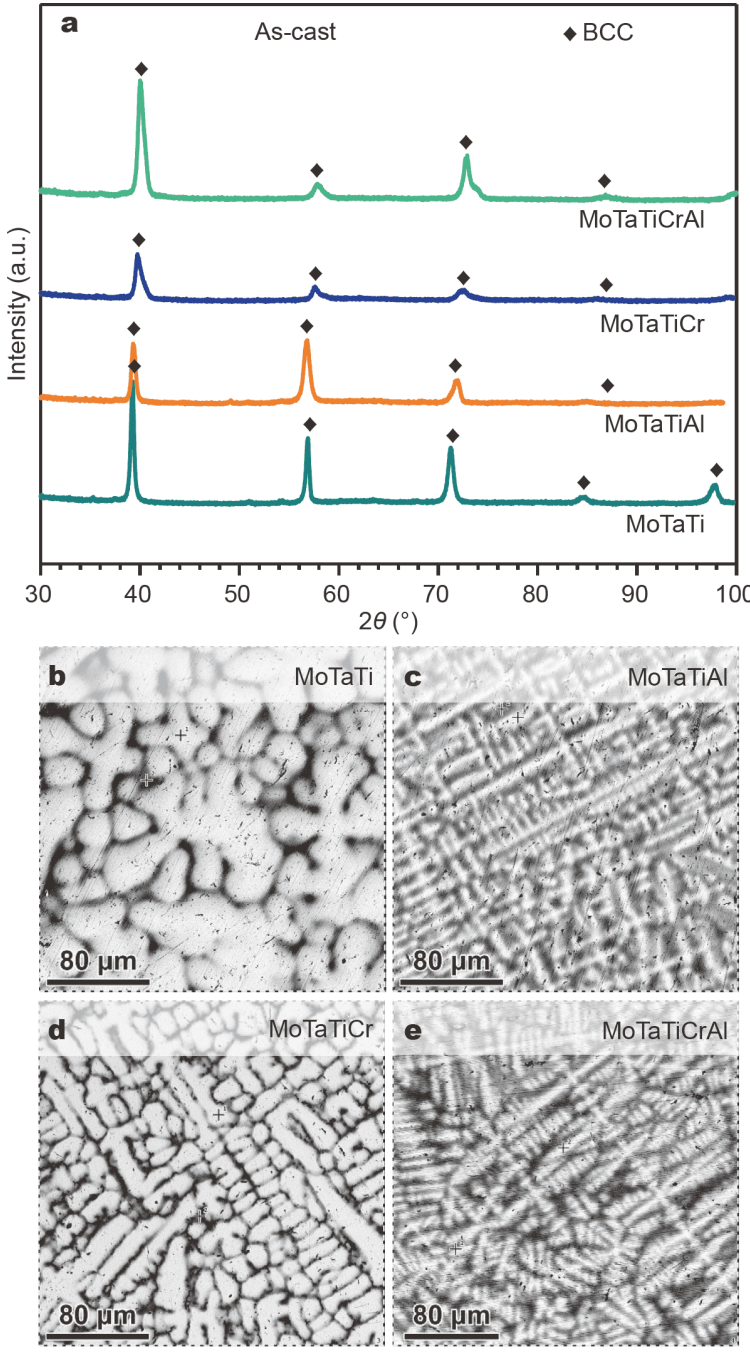

Figure 1 XRD patterns of the studied alloys (a) and microstructures observed by SEM for MoTaTi (b), MoTaTiAl (c), MoTaTiCr (d), and MoTaTiCrAl (e), respectively.

Table 1 Summary of atomic size difference $(\delta)$, mixing enthalpy $\left(\Delta H_{\text {mix }}\right)$, and mixing entropy $\left(\Delta S_{\text {mix }}\right)$ for MoTaTi, MoTaTiAl, MoTaTiCr, and MoTaTiCrAl

\begin{tabular}{ccccc}
\hline & MoTaTi & MoTaTiAl & MoTaTiCr & MoTaTiCrAl \\
\hline$\delta$ & 2.91 & 2.55 & 5.93 & 5.50 \\
$\begin{array}{c}\Delta H_{\text {mix }} \\
\left(\mathrm{kJ} \mathrm{mol}^{1}\right)\end{array}$ & -3.5 & -15.5 & -5.5 & -13.8 \\
$\begin{array}{c}\Delta S_{\text {mix }} \\
\left(\mathrm{J} \mathrm{K}^{-1} \text { mol }^{-1}\right)\end{array}$ & 9.13 & 11.53 & 11.53 & 13.38 \\
\hline
\end{tabular}

becomes irregular at $1000^{\circ} \mathrm{C}$. This demonstrates that MoTaTi underwent severe oxidation so that the entire bulk sample was oxidized at $1000^{\circ} \mathrm{C}$. In comparison to MoTaTi, the MoTaTiCr, MoTaTiAl, and MoTaTiCrAl alloys exhibit much better oxidation resistance at high temperatures. With increasing exposure temperature, only color changes can be seen. This suggests that merely thin oxide layers were formed on their surfaces even at a temperature as high as $1000^{\circ} \mathrm{C}$.

Quantitative characterizations were then performed by measuring the mass change as a function of isothermal exposure time at $1000^{\circ} \mathrm{C}$ in air for $5 \mathrm{~h}$. As shown in Fig. 2b, dramatic mass change can be seen for MoTaTi even after a short exposure time. The tendency continues until the end of test. After $5 \mathrm{~h}$ of exposure, the mass gain is as high as $21.8 \mathrm{mg} \mathrm{cm}^{-2}$, indicating the low oxidation resistance of $\mathrm{MoTaTi}$ at $1000^{\circ} \mathrm{C}$. The behavior is consistent with the sample appearance changes shown in Fig. 2a. Addition of Cr, i.e., MoTaTiCr, substantially decreases the mass changes upon isothermal exposure. Only slight mass gain can be seen in the first hour of isothermal exposure. After that, the mass gain curve reaches to a plateau without obvious increase. At $5 \mathrm{~h}$, the mass gain is as low as $0.93 \mathrm{mg} \mathrm{cm}^{-2}$. Addition of $\mathrm{Al}$, i.e., MoTaTiAl, also decreases the mass changes upon isothermal exposure, and the mass gain after $5 \mathrm{~h}$ is $2.39 \mathrm{mg} \mathrm{cm}^{-2}$, significantly smaller than MoTaTi alloy but larger than that of MoTaTiCr alloy. However, simultaneous incorporation of $\mathrm{Cr}$ and $\mathrm{Al}$ into MoTaTi does not further improve the oxidation resistance in terms of mass gain. As shown in Fig. 2b, although the MoTaTiCrAl shows a similar overall behavior as that of MoTaTiCr, the mass gain at $5 \mathrm{~h}$ is higher $\left(1.39 \mathrm{mg} \mathrm{cm}^{-2}\right)$ than that of MoTaTiCr. This indicates that the MoTaTiCr medium entropy alloy possesses better oxidation resistance than its HEA counterpart. Furthermore, the comparison between MoTaTiCr and MoTaTiAl suggests that the incorporation of $\mathrm{Cr}$ plays a more important role than $\mathrm{Al}$ for the enhancement of oxidation resistance in MoTaTiCrAl.

\section{Formation and microstructure of oxide layer}

Fig. 3 shows the XRD spectra of the surface layers from which oxidation products can be revealed. Fig. 4 presents the cross-section back scattering images of the alloys after isothermal exposure in air at $1000^{\circ} \mathrm{C}$ for $10 \mathrm{~h}$, along with the corresponding chemical analyses by EDX mapping. In the case of MoTaTi, the XRD spectrum and SEM images were taken on a sample oxidized at $900^{\circ} \mathrm{C}$.

The oxides of MoTaTi are mainly composed of $\mathrm{TiO}_{2}$ and $\mathrm{MoTiTa}_{8} \mathrm{O}_{25}$ (Fig. 3). As shown in Fig. 4a, the oxide layer is $\sim 160 \mu \mathrm{m}$ in thickness and appears to be porous. EDX mapping indicates that oxygen distribution in the oxide layer is non-uniform. Chemical analysis along the black line in Fig. 4a indicates that Mo concentration is substantially reduced compared with that in non-oxidized 
Table 2 Compositions (at.\%) of MoTaTi, MoTaTiAl, MoTaTiCr, and MoTaTiCrAl measured by EDX

\begin{tabular}{|c|c|c|c|c|c|c|}
\hline Alloy & Region & Mo & $\mathrm{Ta}$ & $\mathrm{Ti}$ & $\mathrm{Cr}$ & $\mathrm{Al}$ \\
\hline & Overall & 32.17 & 33.62 & 34.21 & - & - \\
\hline \multirow[t]{3}{*}{ MoTaTi } & Bright & 34.80 & 39.35 & 25.86 & - & - \\
\hline & Dark & 29.57 & 23.59 & 46.84 & - & - \\
\hline & Overall & 24.65 & 23.90 & 27.00 & - & 24.45 \\
\hline \multirow[t]{3}{*}{ MoTaTiAl } & Bright & 27.61 & 27.30 & 23.30 & - & 21.79 \\
\hline & Dark & 23.50 & 20.01 & 29.09 & - & 27.40 \\
\hline & Overall & 24.34 & 24.63 & 28.51 & 22.52 & - \\
\hline \multirow[t]{3}{*}{ MoTaTiCr } & Bright & 32.83 & 34.63 & 19.56 & 12.99 & - \\
\hline & Dark & 13.41 & 13.27 & 36.30 & 37.03 & - \\
\hline & Overall & 19.73 & 19.70 & 22.27 & 18.67 & 19.63 \\
\hline \multirow[t]{2}{*}{ MoTaTiCrAl } & Bright & 25.23 & 26.37 & 16.50 & 12.60 & 19.30 \\
\hline & Dark & 18.39 & 18.81 & 20.60 & 17.93 & 24.26 \\
\hline
\end{tabular}

a

as cast $500^{\circ} \mathrm{C} \quad 600^{\circ} \mathrm{C} \quad 700^{\circ} \mathrm{C} 800^{\circ} \mathrm{C} 900^{\circ} \mathrm{C} 1000^{\circ} \mathrm{C}$

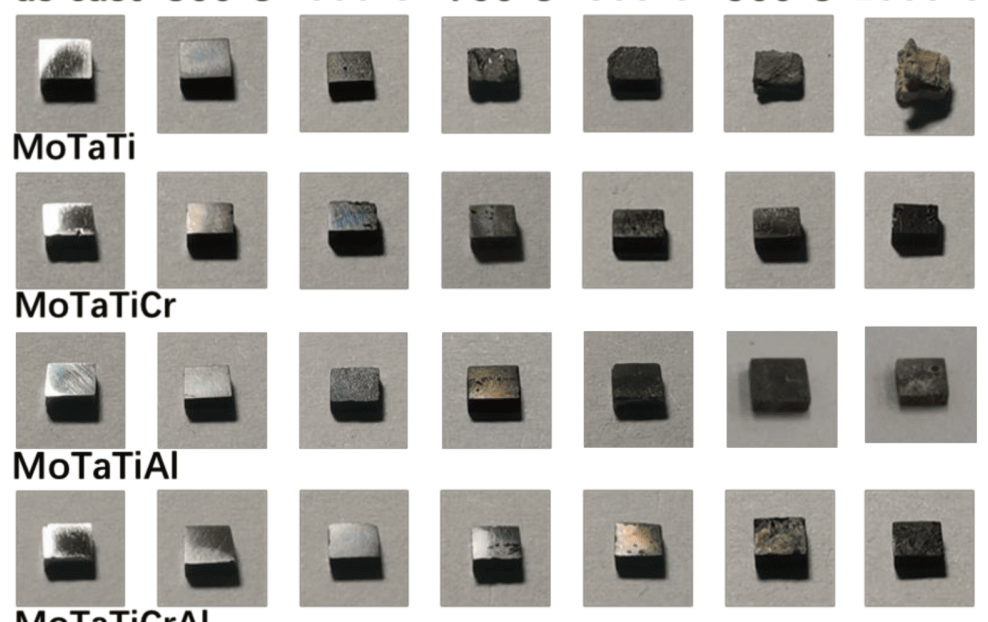

MoTaTiCrAl

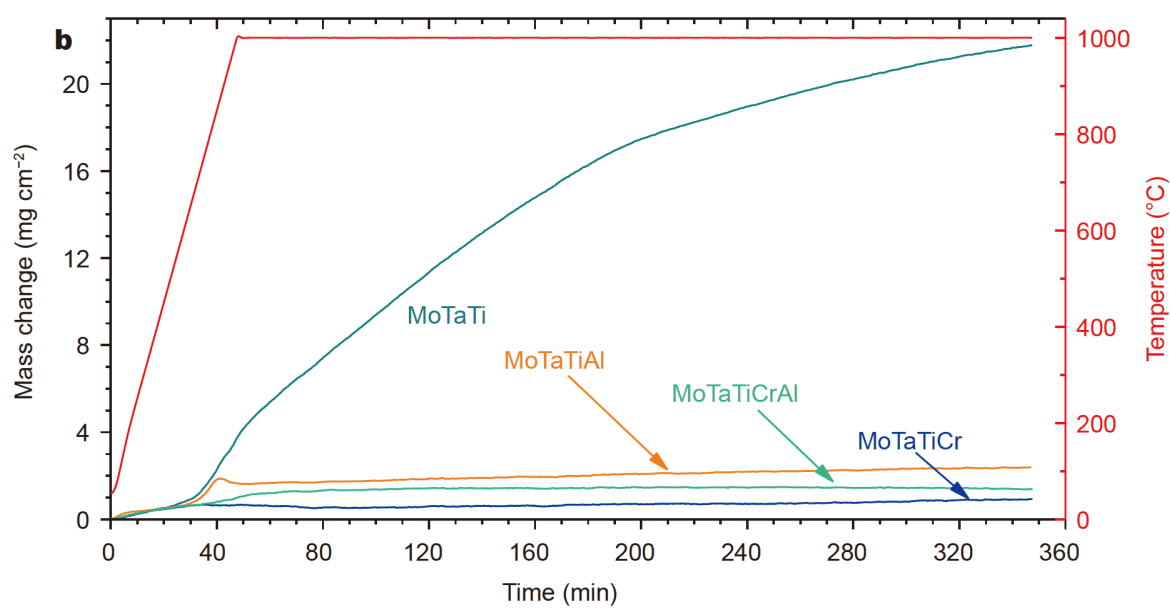

Figure 2 (a) Changes of sample appearances after $10 \mathrm{~h}$ isothermal exposure in air at different temperatures, and (b) mass gain curves measured by TGA at $1000^{\circ} \mathrm{C}$ for MoTaTi, MoTaTiCr, MoTaTiAl, and MoTaTiCrAl alloys. 


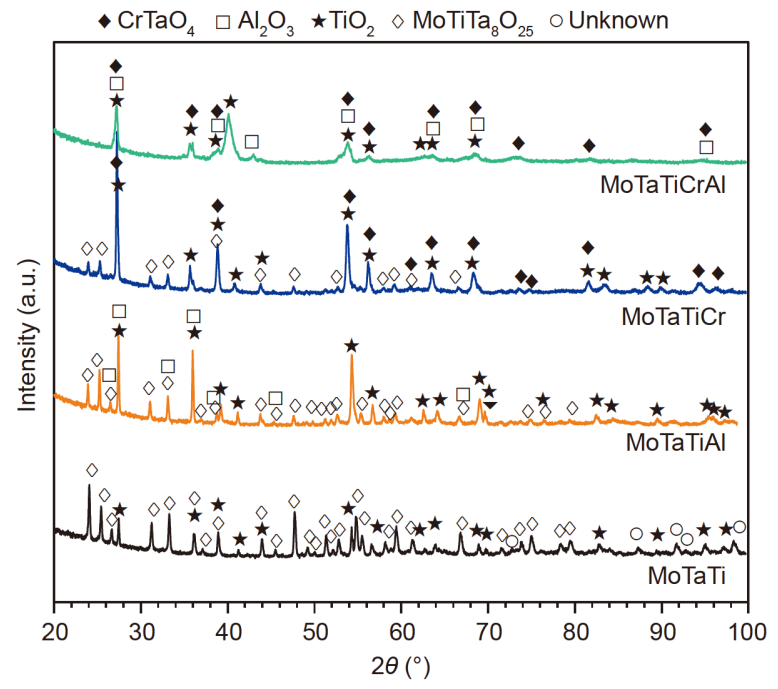

Figure $3 \mathrm{XRD}$ patterns of the surface layers of the studied alloys after isothermal exposure in air for $10 \mathrm{~h}$ at $900^{\circ} \mathrm{C}$ for MoTaTi and $1000^{\circ} \mathrm{C}$ for MoTaTiAl, MoTaTiCr, and MoTaTiCrAl.

region (Fig. 5a, e). This suggests that Mo oxide could have formed but evaporated during oxidation, since $\mathrm{MoO}_{3}$ is highly volatile at temperatures above $800^{\circ} \mathrm{C}[21-23]$. The argument is in consistent with previous investigations [13-16]. Because the evaporation of Mo oxides creates porosity and thus additional pathways for oxygen diffusion, oxidation of region underneath oxide layer cannot be prevented. This explains the severe oxidation of MoTaTi.

XRD analysis on MoTaTiAl indicates the formation of $\mathrm{Al}_{2} \mathrm{O}_{3}$, as well as $\mathrm{TiO}_{2}$ and $\mathrm{MoTiTa}_{8} \mathrm{O}_{25}$ (Fig. 3). The oxide layer has an average thickness of $\sim 35 \mu \mathrm{m}$, which is much less than that in MoTaTi. This is consistent with the better oxidation resistance of MoTaTiAl, and confirms that the addition of $\mathrm{Al}$ into MoTaTi is helpful for the enhancement of oxidation resistance. As revealed by SEM/EDX (Fig. 4b), the microstructure of oxide layer duplicates that of non-oxidized region. However, large porosity can be observed both in the oxide layer and at the wavy interface between oxide layer and non-oxidized alloy. According to EDX mapping (Fig. 4b), the formation of porosity can be ascribed to the evaporation of Mo oxides during oxidation, because the concentration of Mo is significantly reduced in the oxide layer (Fig. 5b and f). It can be seen that there is $\mathrm{Al}$ enrichment at the top of oxide layer, while Ti enrichment is observed at the interface between oxidized and non-oxidized regions. Therefore, it is possible that upon oxidation, $\mathrm{Al}_{2} \mathrm{O}_{3}$ was formed as the top layer, but incapable to form a continuous layer. This argument is further supported by the significant concentration variation of $\mathrm{Al}$ and $\mathrm{Ti}$ in the oxide layer in contrast to the much more uniform distribution of Ta (Fig. 4b). This indicates that even though $\mathrm{Al}$ addition can help to increase oxidation resistance of

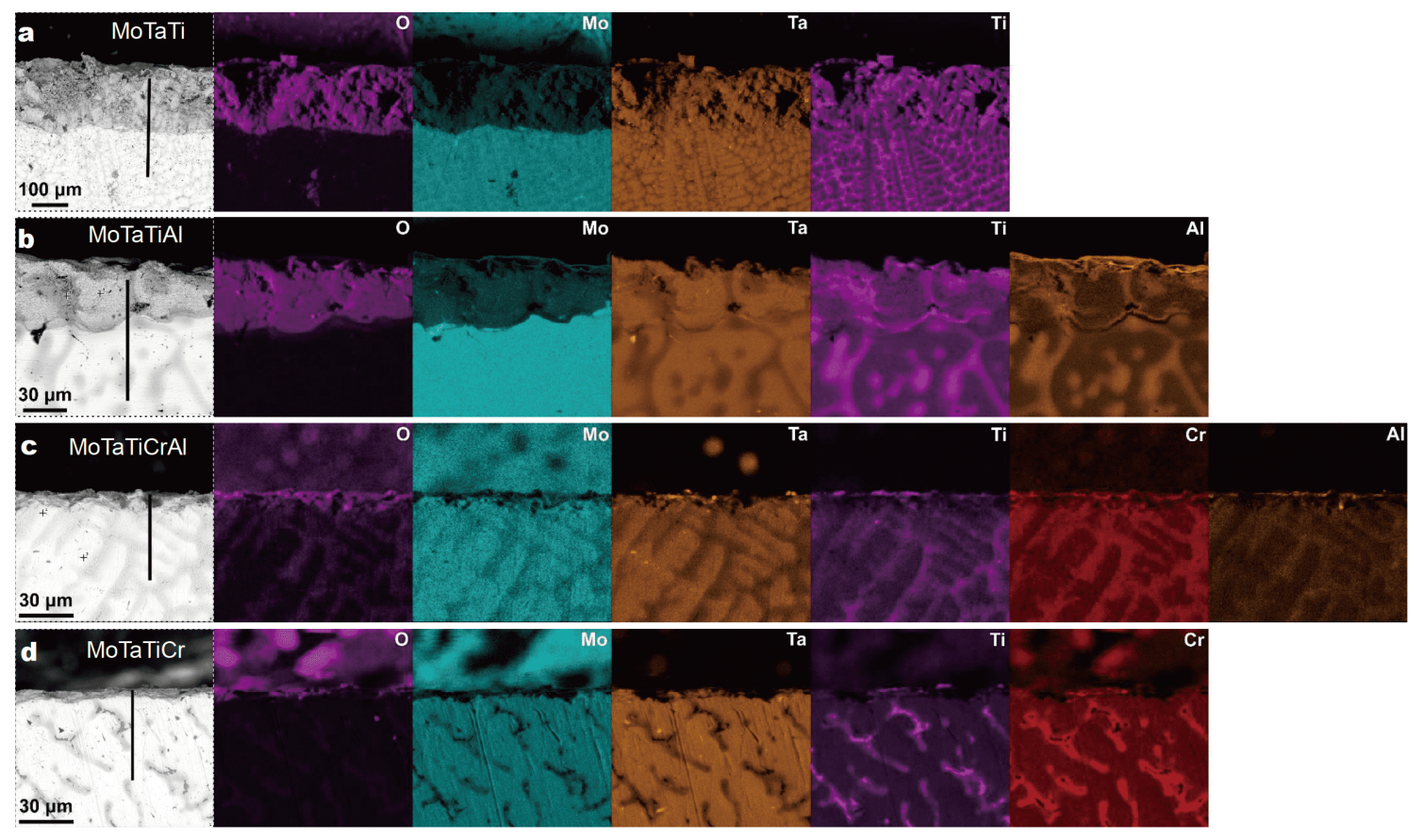

Figure 4 Cross-section images and the corresponding chemical analyses by EDX mappings after isothermal exposure in air for $10 \mathrm{~h}$ at $900^{\circ} \mathrm{C}$ for $\operatorname{MoTaTi}(\mathrm{a})$, and $1000^{\circ} \mathrm{C}$ for MoTaTiAl (b), MoTaTiCrAl (c), and MoTaTiCr (d). 

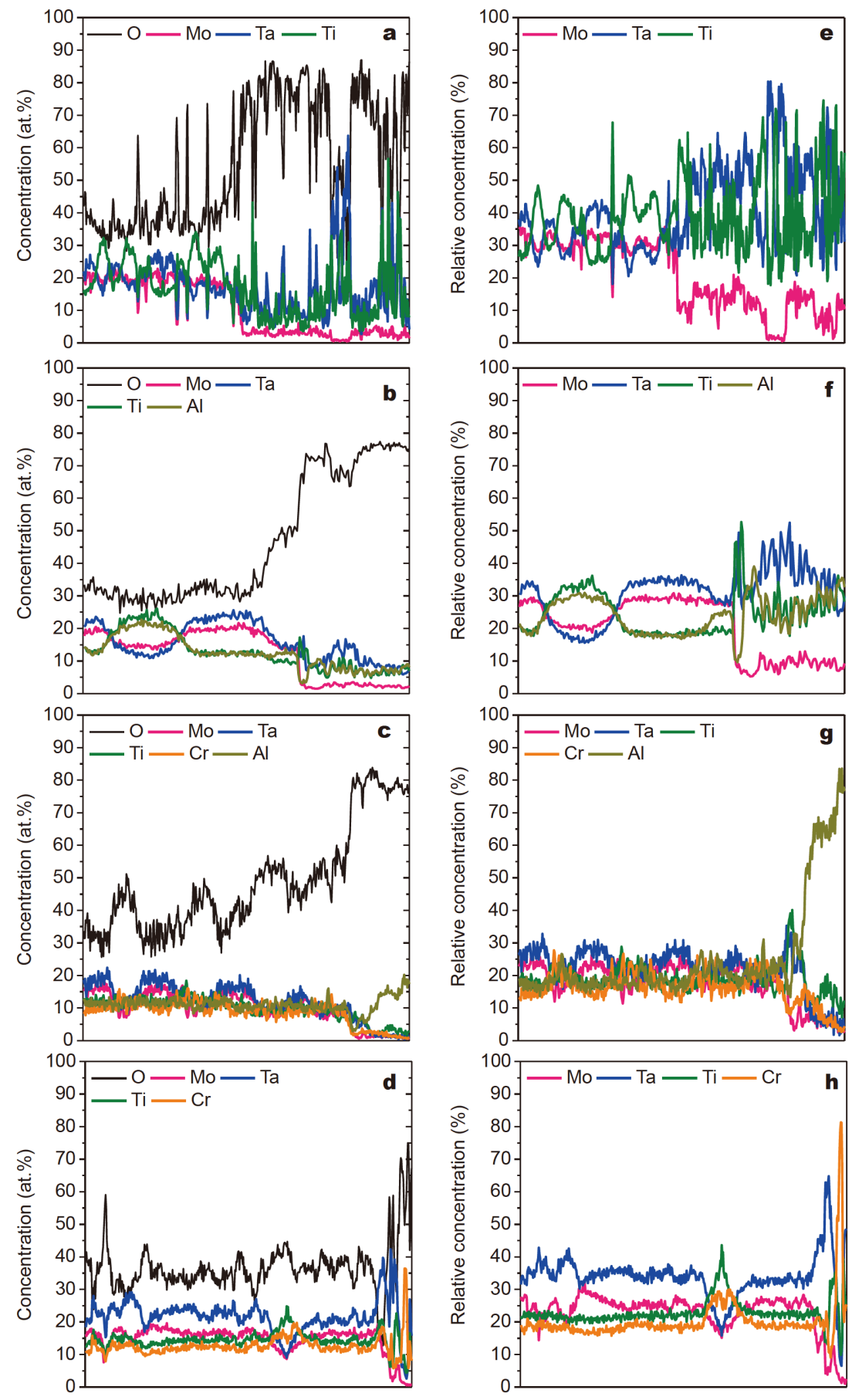

Figure 5 Chemical analyses by EDX line scan along the black line shown in Fig. 4. (a, e) MoTaTi; (b, f) MoTaTiAl; (c, g) MoTaTiCrAl; (d, h) MoTaTiCr. The panels from $(\mathrm{e}-\mathrm{h})$ show the relative concentration of each constituent elements without taking oxygen into account.

MoTaTi, formation of $\mathrm{Al}_{2} \mathrm{O}_{3}$ cannot result in a dense layer as an effective barrier against further oxidation.

As indicated by XRD analysis (Fig. 3), the addition of $\mathrm{Cr}$ into MoTaTiAl results in the formation of $\mathrm{CrTaO}_{4}$ that has been considered effective to prevent oxidation of RHEA by inhibition of outward cation diffusion [14]. Other oxides in MoTaTiCrAl are mainly $\mathrm{TiO}_{2}$ and $\mathrm{Al}_{2} \mathrm{O}_{3}$. As shown in Fig. 4c, the distribution of $\mathrm{Ta}$ and $\mathrm{Cr}$ mat- 
ches quite well, confirming the formation of $\mathrm{CrTaO}_{4}$. However, neither $\mathrm{Ti}$ nor $\mathrm{Al}$ is distributed evenly across the surface, suggesting that they fail to form a continuous layer. In addition, it can be seen that the concentration reduction in Mo (Fig. $5 \mathrm{c}$ and g) creates voids where both $\mathrm{Ta}$ and $\mathrm{Al}$ are depleted (Fig. 4c). This indicates that neither $\mathrm{CrTaO}_{4}$ nor $\mathrm{Al}_{2} \mathrm{O}_{3}$ continuously cover the surface, and the discontinuity leaves pathway for oxygen diffusion into the region underneath the oxide layer. This is supported by the similar distribution of $\mathrm{Ti}$ and $\mathrm{O}$ in the interior of the alloy that implies possible internal oxidation.

Surprisingly, the removal of Al from MoTaTiCrAl to MoTaTiCr results in a continuous $\mathrm{CrTaO}_{4}$ layer (Fig. 3), as confirmed by the coincident distribution of $\mathrm{Cr}, \mathrm{Ta}$, and oxygen. $\mathrm{TiO}_{2}$ and $\mathrm{MoTiTa}_{8} \mathrm{O}_{25}$ have also been identified in MoTaTiCr (Fig. 3). EDX mapping indicates that $\mathrm{TiO}_{2}$ forms in the outer-most layer and mainly in the Ti-rich region of the alloy. This can be attributed to its large thermodynamic driving force of formation than that of $\mathrm{CrTaO}_{4}$ [15]. In the oxide layer, the reduction in Mo concentration still happens but is only significant near the top of the oxide layer (Fig. $5 \mathrm{~d}$ and $\mathrm{h}$ ). This clearly demonstrates that the formation of a continuous $\mathrm{CrTaO}_{4}$ layer is the primary reason for the remarkable oxidation resistance of MoTaTiCr. In addition, the results also indicate that $\mathrm{Al}_{2} \mathrm{O}_{3}$ is not a prerequisite for enhancing oxidation resistance in the MoTaTi- $(\mathrm{Cr}, \mathrm{Al})$ alloy system.

\section{DISCUSSION}

As shown in Fig. 5, there is Mo reduction in the oxide layers in all of the studied alloys, which can be ascribed to the evaporation of Mo oxide that is volatile at high temperature $[14,15,22,23]$. This can be clearly seen from the variation of relative concentration without taking oxygen into account. In the case of MoTaTiCr, the formation of $\mathrm{CrTaO}_{4}$ can effectively suppress the escape of $\mathrm{Mo}$, as the reduction of Mo is only significant at the very top of oxide layer (Fig. 5h) and the relative concentration of Mo quickly becomes the same as that of non-oxidized alloy. For the other three alloys, however, Mo reduction is constant across the entire oxide layer. The reduction of Mo relative to the amount in non-oxidized region is more than $70 \%$. Therefore, it is reasonable to conclude that the escape of Mo upon oxidation leads to underestimated mass gain in TGA measurement, in particular, for MoTaTi, MoTaTiAl, and MoTaTiCrAl.

For the oxidation of metallic alloys, there are two extreme cases that can be expected [16]. In the first case, the oxide fails to form a continuous passive layer due to either oxide evaporation or crack formation induced by volume mismatch between the oxide and the alloy. The discontinuity leads to easy oxidation of the alloy underneath the oxide layer. In the second case, a stable and dense surface oxide layer forms and retards oxygen diffusion through the layer, so that further oxidation is prevented. Hence, the compactness of oxides plays an important role in oxidation of alloys.

The volume change induced by the formation of oxide can be quantified by PBR $[24,25]$, which is the ratio between the volume of oxide formed and the volume of the corresponding alloy, i.e., $\mathrm{PRB}=V_{\text {oxide }} / V_{\text {alloy. }}$. Dense oxide layers are expected if PBR is in the range of 1-2, beyond which the oxide layer can be discontinuous either due to small volume of oxides or the cracking of oxide layer induced by internal stress [24,25].

To gain insight into the origin of the oxidation resistance of MoTaTi-(Cr, Al) alloys, we estimated PBR of oxides observed from XRD analyses. In the estimation, the volumes occupied by each atom in the alloys are considered the same. In BCC lattice, the volume of every 2 atoms could be written as $a^{3}$ with $a$ the lattice constant, and PBR can be written as [24]: $\mathrm{PRB}=V_{\text {oxide }} / 0.5 z a^{3}$, where $V_{\text {oxide }}$ is the volume of oxide, $z$ is the number of metallic atoms in one oxide cell. The estimated PBR values are summarized in Table 3. Apparently, $\mathrm{MoTiTa}_{8} \mathrm{O}_{25}$ has a too large PRB value to form a protective layer. This explains why MoTaTi can be easily oxidized. Although $\mathrm{Al}_{2} \mathrm{O}_{3}$ and $\mathrm{TiO}_{2}$ show ideal $\mathrm{PRB}$, they do not form a continuous layer, leading to the thick oxidized layer in MoTaTiAl. As mentioned above, oxidation in the alloy system is accompanied with evaporation of Mo oxide. This leaves voids and pores in the oxide layer composed of the mixture of different oxides. Such voids may cause break of originally protective $\mathrm{Al}_{2} \mathrm{O}_{3}$ that has a small PRB value. To compensate the volume mismatch between the oxide layer and non-oxidized alloy, the oxide of properly larger PRB is required. It is thus reasonable to assume that the formation of $\mathrm{CrTaO}_{4}$ in $\mathrm{MoTaTiCr}$ plays such a role, so that a continuous and protective layer quickly forms. This assumption is supported by the fact that the reduction of Mo occurs mainly near the top of oxide layer (Fig. 5h).

Table 3 Calculated PBR for the oxides formed in the alloys

\begin{tabular}{ccccc}
\hline Alloy & $\mathrm{MoTiTa}_{8} \mathrm{O}_{25}$ & $\mathrm{TiO}_{2}$ & $\mathrm{CrTaO}_{4}$ & $\mathrm{Al}_{2} \mathrm{O}_{3}$ \\
\hline MoTaTi & 2.80 & 1.86 & - & - \\
MoTaTiCr & 2.92 & 1.94 & 2.01 & - \\
MoTaTiAl & 2.84 & 1.90 & - & 1.24 \\
MoTaTiCrAl & 2.95 & 1.96 & 2.02 & 1.29 \\
\hline
\end{tabular}




\section{CONCLUSION}

In summary, we have fabricated MoTaTi, MoTaTiCr, MoTaTiAl and MoTaTiCrAl alloys by arc melting. All of the as-cast alloys exhibit a single-phase solid solution of BCC structure. Oxidation resistance of the MoTaTi- $(\mathrm{Cr}$, $\mathrm{Al}$ ) alloys was evaluated by isothermal exposure at high temperatures in combination with thermogravimetric analyses. It is found that $\mathrm{Al}$ addition is not a prerequisite for enhanced oxidation resistance of the $\operatorname{MoTaTi}-(\mathrm{Cr}, \mathrm{Al})$ alloy system. Instead, MoTaTiCr is most resistant to oxidation among the four alloys. Microstructural characterizations by XRD and SEM/EDX suggest that the enhanced oxidation resistance of MoTaTiCr arises from the formation of $\mathrm{CrTaO}_{4}$ that can compensate the volume mismatch between the oxide layer and non-oxidized alloy due to the escape of Mo oxide. The outstanding oxidation resistance of MoTaTiCr along with its remarkable mechanical properties [10] makes it a promising candidate for high-temperature applications.

Received 6 March 2020; accepted 5 April 2020;

published online 9 June 2020

1 Yamamoto Y, Brady MP, Lu ZP, et al. Creep-resistant, $\mathrm{Al}_{2} \mathrm{O}_{3}$ forming austenitic stainless steels. Science, 2007, 316: 433-436

2 Huntz AM, Bague V, Beauplé G, et al. Effect of silicon on the oxidation resistance of $9 \%$ Cr steels. Appl Surf Sci, 2003, 207: 255275

3 Liu Z, Gao W, Dahm K, et al. The effect of coating grain size on the selective oxidation behaviour of $\mathrm{Ni}-\mathrm{Cr}-\mathrm{Al}$ alloy. Scripta Mater, 1997, 37: 1551-1558

4 Miracle DB, Senkov ON. A critical review of high entropy alloys and related concepts. Acta Mater, 2017, 122: 448-511

5 Cantor B, Chang ITH, Knight P, et al. Microstructural development in equiatomic multicomponent alloys. Mater Sci Eng-A, 2004, 375-377: 213-218

6 Ye YF, Wang Q, Lu J, et al. High-entropy alloy: Challenges and prospects. Mater Today, 2016, 19: 349-362

7 Yeh JW, Chen SK, Lin SJ, et al. Nanostructured high-entropy alloys with multiple principal elements: Novel alloy design concepts and outcomes. Adv Eng Mater, 2004, 6: 299-303

8 Zhang Y, Zuo TT, Tang Z, et al. Microstructures and properties of high-entropy alloys. Prog Mater Sci, 2014, 61: 1-93

9 Gludovatz B, Hohenwarter A, Catoor D, et al. A fracture-resistant high-entropy alloy for cryogenic applications. Science, 2014, 345: $1153-1158$

10 Coury FG, Kaufman M, Clarke AJ. Solid-solution strengthening in refractory high entropy alloys. Acta Mater, 2019, 175: 66-81

11 Senkov ON, Wilks GB, Miracle DB, et al. Refractory high-entropy alloys. Intermetallics, 2010, 18: 1758-1765

12 Senkov ON, Miracle DB, Chaput KJ, et al. Development and exploration of refractory high entropy alloys-A review. J Mater Res, 2018, 33: 3092-3128

13 Gorr B, Müller F, Azim M, et al. High-temperature oxidation behavior of refractory high-entropy alloys: Effect of alloy composition. Oxid Met, 2017, 88: 339-349
14 Lo KC, Chang YJ, Murakami H, et al. An oxidation resistant refractory high entropy alloy protected by $\mathrm{CrTaO}_{4}$-based oxide. Sci Rep, 2019, 9: 7266

15 Müller F, Gorr B, Christ HJ, et al. On the oxidation mechanism of refractory high entropy alloys. Corrosion Sci, 2019, 159: 108161

16 Senkov ON, Senkova SV, Dimiduk DM, et al. Oxidation behavior of a refractory $\mathrm{NbCrMo}_{0.5} \mathrm{Ta}_{0.5} \mathrm{TiZr}$ alloy. J Mater Sci, 2012, 47: 6522-6534

17 Senkov ON, Miller JD, Miracle DB, et al. Accelerated exploration of multi-principal element alloys with solid solution phases. Nat Commun, 2015, 6: 6529

18 Guo S, Hu Q, Ng C, et al. More than entropy in high-entropy alloys: Forming solid solutions or amorphous phase. Intermetallics, 2013, 41: 96-103

19 Zhang Y, Zhou Y, Lin J, et al. Solid-solution phase formation rules for multi-component alloys. Adv Eng Mater, 2008, 10: 534-538

20 Sohn S, Liu Y, Liu J, et al. Noble metal high entropy alloys. Scripta Mater, 2017, 126: 29-32

21 Mendiratta MG, Parthasarathy TA, Dimiduk DM. Oxidation be-

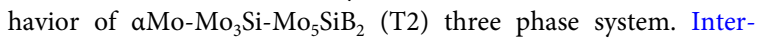
metallics, 2002, 10: 225-232

22 Gulbransen EA. High-temperature oxidation of tungsten, molybdenum and carbon. Nature, 1963, 198: 82-83

23 Gulbransen EA, Andrew KF, Brassart FA. Oxidation of molybdenum $550^{\circ} \mathrm{C}$ to $1700^{\circ} \mathrm{C}$. J Electrochem Soc, 1963, 110: 952-959

24 Xu C, Gao W. Pilling-bedworth ratio for oxidation of alloys. Mater Res Innovations, 2000, 3: 231-235

25 Dunn JS. The high temperature oxidation of metals. Proc R Soc Lond A, 1926, 111: 203-209

Acknowledgements This work was supported by the National Key Research and Development Program of China (2018YFA0703600), the National Science Fund for Distinguished Young Scholars (51825104), the Key Research Program of Frontier Sciences of Chinese Academy of Sciences (QYZDY-SSW-JSC017), the Strategic Priority Research Program of Chinese Academy of Sciences (XDB30000000), the Key Basic and Applied Research Program of Guangdong Province, China (2019B030302010), and the National Natural Science Foundation of China (11790291 and 61888102).

Author contributions Liu YH conceived the research. Li LC, Li MX, and Liu YH designed the experiments. Li LC performed the experiments with assistance from Liu M and Sun BY. Li LC, Liu YH, Wang C analyzed the data. Li LC and Liu YH wrote the paper with input from Wang C, Li MX, Huo JT and Wang WH. All authors contributed to the general discussion.

Conflict of interest The authors declare no competing interest.

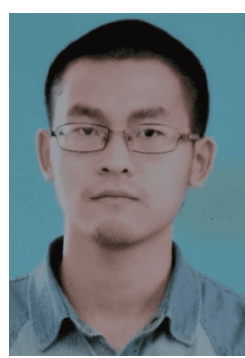

Li-Chao $\mathbf{~ L i}$ is currently a $\mathrm{PhD}$ candidate in condensed matter physics under the supervision of Professor Yan-Hui Liu at the Institute of Physics, Chinese Academy of Sciences. He received his BSc degree in physics from Lanzhou University in 2015. His research focuses on the phase formation and properties of high entropy alloys. 


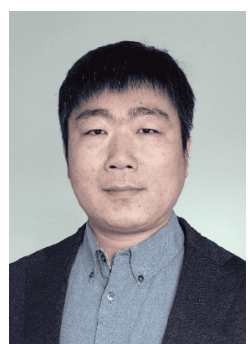

Yan-Hui Liu received his $\mathrm{PhD}$ degree from the Institute of Physics, Chinese Academy of Sciences. From 2007 to 2016, he conducted research in Tohoku University (Japan) and Yale University (USA). He joined the Institute of Physics, Chinese Academy of Sciences as an associate professor in 2016, and was promoted to professor in 2018. His research focuses on the development of high-performing alloys, in particular, metallic glasses and high-entropy alloys, by combinatorial approaches.
通过去除Al元素提高MoTaTiCrAl高熵合金的抗 氧化性

李力超 ${ }^{1,2}$, 李明星 ${ }^{1,3}$, 刘明 ${ }^{1,6}$, 孙博阳 ${ }^{1,2}$, 王超 ${ }^{1}$, 霍军涛 ${ }^{1,5,7}$, 汪卫华 ${ }^{1,3,4,5}$, 柳延辉 $1,3,4,5^{*}$

摘要 难熔高熵合金具有高温应用所需的优异力学性能, 但其高温 抗氧化性却很少受到关注. 近期的研究表明, MoTaTiCrAl合金呈现 较好的抗氧化性, 但 $\mathrm{Al}$ 和 $\mathrm{Cr}$ 两种元素对提高抗氧化性的作用仍未 得以阐明. 本文发现, $\mathrm{Al}$ 元素对防止MoTaTiCrAl合金的氧化是不 必要的, 去除 $\mathrm{Al}$ 元素反而能够获得更抗氧化的MoTaTiCr中熵合金. 结构表征和成分分析表明, MoTaTiCr合金的抗氧化性主要是因为 合金表面能够形成连续的 $\mathrm{CrTaO}_{4}$ 氧化层. 本文结果说明, 除了采用 $\mathrm{Al}_{2} \mathrm{O}_{3}, \mathrm{Cr}_{2} \mathrm{O}_{3}, \mathrm{SiO}_{2}$ 等典型氧化物作为保护层, 在合金表面生成复杂 氧化物也能有效提升难熔高熵合金的抗氧化性能. 